\title{
INVERSE CLUSTER SETS
}

\author{
T. R. HAMLETT AND PAUL E. LONG
}

ABSTRACT. For a function $f: X \rightarrow Y$, the cluster set of $f$ at $x \in X$ is the set of all $y \in Y$ such that there exists a filter $f$ on $X$ converging to $x$ and the filter generated by $f(\mathfrak{F})$ converges to $y$. The inverse cluster set of $f$ at $y \in Y$ is the set of all $x \in X$ such that $y$ belogngs to the cluster set of $f$ at $x$. General properties of inverse cluster sets are proved, including a necessary and sufficient condition for continuity. Necessary and sufficient conditions for functions to have a closed graph in terms of inverse cluster sets are also given. Finally, a known the orem giving a condition as to when a connected function is also a connectivity function is generalized and further investigated in terms of inverse cluster sets.

1. Introduction. The idea of defining an inverse cluster set arises from the concept of a cluster set as found in [7] and [4] as well as elsewhere. The cluster set of a function $f: X \rightarrow Y$ at $x \in X$ is the set of all $y \in Y$ such that there exists a filter $\mathcal{F}$ on $X$ converging to $x$ and the filter generated by $f(\mathcal{F})$ converges to $y$. We define the inverse cluster set of $f$ at $y \in Y$ to be the set of all $x \in X$ such that $y$ belongs to the cluster set of $f$ at $x$. After discussing some general properties of inverse cluster sets, their relationship to functions with closed graphs as well as connectedness is investigated.

Throughout, we use $\Re(x)$ to denote the neighborhood system at the point $x$. If $f: X \rightarrow Y$ is a function and $\mathcal{F}$ is a filter on $X$, the filterbase $f(\mathcal{F})$ generates a filter which we also denote by $f(\mathcal{F})$. The graph of a function $f$ : $X \rightarrow Y$ is denoted by $G(f)=\{(x, f(x)): x \in X\}$. For the set $A, \mathrm{Cl}(A)$ denotes the closure of $A$.

2. Basic properties of inverse cluster sets.

2.1. Definition [4]. Let $f: X \rightarrow Y$ be any function. Then $y \in Y$ is an element of the cluster set of $f$ at $x$, denoted by $\mathcal{C}(f ; x)$, if there exists a filter $\mathcal{F}$ on $X$ such that $\mathcal{F}$ converges to $x$ and $f(\mathcal{F})$ converges to $y$.

2.2. Jefinition. Let $f: X \rightarrow Y$ be any function. The inverse cluster set of $f$ at $y \in Y$, denoted by $\mathcal{P}^{-1}(f ; y)$, is the set of all $x \in X$ such that $y \in \mathcal{C}(f ; x)$.

2.3. Theorem. Let $f: X \rightarrow Y$ be a function. Then the following are equivalent:

Received by the editors December 9, 1974 .

AMS (MOS) subject classifications (1970). Primary 54A20; Secondary 54C10. 
(1) $x \in \mathcal{C}^{-1}(f ; y)$.

(2) $x \in \bigcap\left\{\mathrm{Cl}\left(f^{-1}(V): V \in \mathcal{N}(y)\right\}\right.$.

(3) The filterbase $f^{-1}(\mathcal{H}(y))$ accumulates to $x$.

(4) $y \in \bigcap\{\mathrm{Cl}(f(U)): U \in \mathcal{Y}(x)\}$.

(5) $f(\mathcal{H}(x))$ accumulates to $y$.

(6) There exists a net $x_{a} \rightarrow x$ such that $f\left(x_{a}\right) \rightarrow y$.

Proof. Theorem 2.2 of [4] states that $y \in \mathcal{C}(f ; x)$ if and only if $f^{-1}(\Re(y))$ accumulates at $x$ if and only if $y \in \bigcap\{C l(f(U)): U \in \Re(x)\}$. The conditions of the theorem then follow in a straightforward manner.

2.4. Corollary. For $f: X \rightarrow Y$, the set $\mathcal{C}^{-1}(f ; y)$ is closed for every $y \in Y$.

2.5. Corollary. If $f: X \rightarrow Y$ is a given function, then $\mathrm{Cl}\left(f^{-1}(y)\right) \subset$ $\mathcal{C}^{-1}(f ; y)$ for every $y \in Y$.

Proof. Condition (2) of Theorem 2.3.

2.6. Theorem. Let $X$ be compact and $f: X \rightarrow Y$ a function such that $\mathrm{Cl}(f(X))=Y$. Then $\mathcal{C}^{-1}(f ; y) \neq \varnothing$ for every $y \in Y$.

Proof. For each $y \in Y, f^{-1}(\mathcal{H}(y))$ is a filterbase on the compact $X$, hence must have an accumulation point.

Evidently, the set $\mathcal{C}^{-1}(f ; y)$ need not be connected even for continuous functions. In our efforts to find a condition under which $\mathcal{C}^{-1}(f ; y)$ is connected, we use the following definition:

2.7. Definition. The function $f: X \rightarrow Y$ is inverse connected if $f^{-1}(C)$ is connected for every connected $C \subset Y$.

A sufficient condition for $f: X \rightarrow Y$ to be inverse connected, for example, is that $f$ be closed and monotone [6, Theorem 2].

2.8. Theorem. Let $f: X \rightarrow Y$ be inverse connected, $\mathrm{Cl}(f(X))=Y, X$ compact Hausdorff and $Y$ locally connected. Then $\mathcal{C}^{-1}(f ; y)$ is a nonempty continuum for every $y \in Y$.

Proof. By Theorem 2.6, $\mathcal{C}^{-1}(f ; y) \neq \varnothing$. Now let $\mathcal{K}(y)$ be a neighborhood base of connected sets at $y$. It then follows from Theorem 2.3(2) that $\mathcal{C}^{-1}(f ; y)=\bigcap\left\{\mathrm{Cl}^{-1}\left(f^{-1}(V)\right): V \in \mathcal{K}(y)\right\}$. Since $\left\{\mathrm{Cl}\left(f^{-1}(V)\right): V \in \mathcal{K}(y)\right\}$ is a collection of continua directed by inclusion, their intersection is a continuum [8, Theorem 28.2].

2.9. Theorem. Let $f: X \rightarrow Y$ be any function where $Y$ is compact Hausdorff. Then $f$ is continuous at $x_{0} \in X$ if and only if $x_{0} \in \mathcal{C}^{-1}(f ; y)$ for exactly one $y \in Y$.

Proof. Suppose first that $f$ is continuous at $x_{0}$. Then $f\left(\mathcal{N}\left(x_{0}\right)\right) \rightarrow f\left(x_{0}\right)$ 
and, since $Y$ is Hausdorff, $f\left(\mathcal{R}\left(x_{0}\right)\right)$ cannot accumulate to any other point. Now suppose $x_{0} \in \mathrm{C}^{-1}(f ; y)$ for exactly one $y \in Y$. Assume $f$ is not continuous at $x_{0}$. Then there exists an open $V$ containing $y$ such that $f(U) \cap(Y-V) \neq \varnothing$ for every $U \in \Re\left(x_{0}\right)$. Thus, $f\left(\Re\left(x_{0}\right)\right)$ accumulates to some $y \in Y-V$ so that $y \in \mathcal{C}\left(f ; x_{0}\right)$ which implies $x_{0} \in \mathcal{C}^{-1}(f ; y)$. But $x_{0} \in$ $\mathcal{C}^{-1}\left(f ; f\left(x_{0}\right)\right)$ also, and since $y \neq f\left(x_{0}\right)$, we have a contradiction to our hypothesis. It follows that $f$ is continuous at $x_{0}$.

2.10. Theorem [4]. Let $f: X \rightarrow Y$ be a connected function, $X$ locally connected and $Y$ compact Hausdorff. Then $f$ is continuous at $x_{0} \in X$ if and only if $\left\{y: x_{0} \in \mathcal{C}^{-1}(f ; y)\right\}$ is countable.

2.11. Theorem. Let $f: X \rightarrow Y$ be surjective. If $\mathcal{C}^{-1}(f ; y)$ is degene. rate for every $y \in Y$, then $X$ is a $T_{1}$-space and $f$ is a bijection.

Proof. Theorem 2.4 shows each point in $X$ is closed so that $X$ is a $T_{1}$-space. If $f\left(x_{1}\right)=f\left(x_{2}\right)=y$, then $\left\{x_{1}, x_{2}\right\} \subset \mathcal{C}^{-1}(f ; y)$. The hypothesis now implies $x_{1}=x_{2}$ so that $f$ is injective.

2.12. Theorem. If $f: X \rightarrow Y$ is a bijection, then $\mathcal{C}^{-1}(f ; y)=\mathcal{C}\left(f^{-1} ; y\right)$.

Proof. Definition 2.1 and Theorem 2.2 of [4] give $\mathcal{C}^{-1}(f ; y)=$ $\bigcap\left\{\mathrm{Cl}\left(f^{-1}(V)\right): V \in \mathcal{T}(y)\right\}=\mathcal{C}\left(f^{-1} ; y\right)$.

2.13. Theorem. Let $f: X \rightarrow Y$ be surjective and inverse connected where $X$ is compact Hausdorff and $Y$ is locally connected. If $\mathrm{C}^{-1}(f ; y)$ is countable for every $y \in Y$, then

(1) $f$ is a bijection, and

(2) $f^{-1}$ is continuous.

Proof. By Theorem 2.8, $\mathcal{C}^{-1}(f ; y)$ is a nonempty continuum for every $y \in Y$ and the hypothesis that $\mathcal{C}^{-1}(f ; y)$ is countable implies $\mathcal{C}^{-1}(f ; y)$ is a singleton. It now follows from Theorem 2.11 that $f$ is a bijection.

By Theorem 2.12, $\mathcal{C}^{-1}(f ; y)=\mathcal{C}\left(f^{-1} ; y\right)$ is degenerate for every $y \in \mathrm{Y}$. Since $X$ is compact Hausdorff, $f^{-1}$ is continuous by Theorem 2.3 of [4].

3. Inverse cluster sets and the closed graph.

3.1. Theorem. Let $f: X \rightarrow Y$ be any function and let $y \in Y$. Then $(x, y) \in X \times Y$ is a cluster point of $G(f)$ that does not belong to $G(f)$ if and only if $x \in \mathcal{C}^{-1}(f ; y)-f^{-1}(y)$.

3.2. Theorem. For $f: X \rightarrow Y, G(f)$ is closed if and only if $\mathcal{C}^{-1}(f ; y)$ $=f^{-1}(y)$ for every $y \in Y$.

Proof. Since $\mathcal{C}^{-1}(f ; y)=f^{-1}(y)$ if and only if $\mathcal{C}^{-1}(f ; y)-f^{-1}(y)=\varnothing$. Theorem 3.1 gives the desired result. 
3.3. Theorem. Let $f: X \rightarrow Y$ be closed and $X$ regular. Then $\mathcal{C}^{-1}(f ; y)$ $=\mathrm{Cl}\left(f^{-1}(y)\right)$ for every $y \in Y$.

Proof. Since $\mathrm{Cl}\left(f^{-1}(y)\right) \subset \mathcal{C}^{-1}(f ; y)$ for every $y \in Y$, we need only show the reverse inclusion. Suppose there exists a point $x \in X$ such that $x \in$ $\mathcal{C}^{-1}(f ; y)-\mathrm{Cl}\left(f^{-1}(y)\right)$. The regularity of $X$ then assures the existence of disjoint open sets $U$ and $V$ containing $x$ and $\mathrm{Cl}\left(f^{-1}(y)\right)$, respectively. Now using the fact that $f$ is closed, there exists an open $W$ containing $y$ such that $f^{-1}(W) \subset V\left[2\right.$, Theorem 11.2, p. 86]. Hence, $x \notin \mathrm{Cl}\left(f^{-1}(W)\right)$ which implies $x \notin \mathcal{C}^{-1}(f ; y)$. This contradiction gives $\left.\mathcal{C}^{-1}(f ; y) \subset \mathrm{Cl}^{-1}(y)\right)$ and establishes the theorem.

The following Corollary shows how one of Fuller's results [3] may be proved using inverse cluster sets.

3.4. Corollary [3, Corollary 3.9]. Let $f: X \rightarrow Y$ be closed and $X$ regular. If $f^{-1}(y)$ is closed for every $y \in Y$, then $f$ has a closed graph.

Proof. Theorems 3.3 and 3.2.

3.5. Theorem. Let $f: X \rightarrow Y$ be closed and monotone where $X$ is regular. Then $\mathcal{C}^{-1}(f ; y)$ is connected for every $y \in Y$.

Proof. By Theorem 3.3, $\mathrm{C}^{-1}(f ; y)=\mathrm{Cl}\left(f^{-1}(y)\right)$ for every $y \in Y$. Thus, $e^{-1}(f ; y)$ is the closure of the connected set $f^{-1}(y)$, hence connected.

We have seen that for a given function $f: X \rightarrow Y, \mathcal{C}^{-1}(f ; y)$ is closed for every $y \in Y$. The following definition is used to determine a sufficient condition for a union of such sets to remain closed.

3.6. Definition. Let $f: X \rightarrow Y$ and let $A \subset Y$. Then $\mathcal{C}^{-1}(f ; A)=$ $\bigcup\left\{\mathcal{C}^{-1}(f ; a): a \in A\right\}$.

3.7. Theorem. Let $f: X \rightarrow Y$. If $A \subset Y$ is compact, then $\mathcal{C}^{-1}(f ; A)$ is closed.

Proof. First observe that

$$
\left.\mathcal{C}^{-1}(f ; A)=\bigcup\left\{\mathcal{C}^{-1}(f ; a): a \in A\right\} \subset \bigcap\left\{\mathrm{Cl}^{-1}(V)\right): V \text { open and } A \subset V\right\} \text {. }
$$

We now show the reverse inclusion. Let $x \in \bigcap\left\{\mathrm{Cl}\left(f^{-1}(V)\right): V\right.$ open and $A \subset V\}$ and assume that for all $a \in A$ the filterbase $f^{-1}(\Re(a))$ does not accumulate to $x \in X$. Then for each $a \in A$ there exists a $V(a) \in \Re(a)$ and a $U_{a} \in \Re(x)$ such that $f^{-1}(V(a)) \cap U_{a}=\varnothing$. Now let $\left\{V\left(a_{i}\right): 1 \leq i \leq n\right\}$ be a finite subcollection of $\{V(a): a \in A\}$ which covers $A$ and let $\left\{U_{a(i)}: 1 \leq i \leq n\right\}$ be the corresponding neighborhoods of $x$. It follows that

$$
\bigcap\left\{U_{a(i)}: 1 \leq i \leq n\right\} \cap f^{-1}\left(\bigcup\left\{V\left(a_{i}\right): 1 \leq i \leq n\right\}\right)=\varnothing
$$

so that $x \notin \bigcap\left\{\mathrm{Cl}^{-1}(V)\right): V$ open and $\left.A \subset V\right\}$. But this contradicts our 
hypothesis. We conclude $x \in \mathcal{C}^{-1}(f ; A)$ and this implies $\mathcal{C}^{-1}(f ; A)=$ ก\{Cl $\left(f^{-1}(V)\right): V$ open and $\left.A \subset V\right\}$.

The following Corollary again shows how one of Fuller's results [3] may be obtained using inverse cluster sets.

3.8. Corollary [3, Theorem 3.6]. Let $f: X \rightarrow Y$ be a given function with closed graph. If $A \subset Y$ is compact, then $f^{-1}(A)$ is closed.

Proof. Theorem 3.2 along with Theorem 3.7 shows that

$$
f^{-1}(A)=\bigcup\left\{f^{-1}(a): a \in A\right\}=\bigcup\left\{\mathcal{O}^{-1}(f ; a): a \in A\right\}=\mathcal{\varrho}^{-1}(f ; A) .
$$

3.9. Theorem. Let $f: X \rightarrow Y$ be continuous from the H-closed space $X$ into the Hausdorff space $Y$. Then $f$ maps regular-closed sets onto closed sets.

Proof. Let $M$ be a regular-closed subset of $X$. It follows that $M$ is an $H$-closed subspace of $X$. Now consider any $y \in \mathrm{Cl}(f(M))$. Since $f^{-1}(\Re(y))$ is an open filterbase with a trace on $M, f^{-1}(\mathcal{\gamma}(y))$ accumulates to some $x$ $\in M$ [1, Theorem 3.2] so that by Theorem 2.1(3), $x \in \mathcal{C}^{-1}(f ; y)$. The fact that $f$ has a closed graph, along with Theorem 2.2, implies $x \in \mathcal{C}^{-1}(f ; y)$ $=f^{-1}(y)$ so that $y \in f(M)$. We conclude $f(M)$ is closed.

4. Connectivity functions and inverse cluster sets. For a given function $f: X \rightarrow Y$ where both $X$ and $Y$ are first countable, the inverse cluster set $\mathcal{C}^{-1}(f ; y)$ is precisely the set $T(f ; y)$ as defined in [5, Definition 3.2]. We now show how cluster sets may be used to generalize Theorem 3.6 of [5] after recalling that a connected function $f: X \rightarrow Y$ is one that preserves connected sets and a connectivity function is one such that the induced function $g: X \rightarrow X \times Y$, defined by $g(x)=(x, f(x))^{\prime}$, is connected.

4.1. Theorem. Let $f: X \rightarrow Y$ be a connected function where $X$ is compact. Then $f$ is a connectivity function if for each connected $M \subset X$ and any $x \in M, \mathcal{C}^{-1}(f ; f(x)) \cap \mathrm{Cl}(M)=\{x\}$.

Proof. Let $f$ be connected and assume the given condition. Suppose there exists a connected $M \subset X$ such that $g(M)=H \cup K$ where $H$ and $K$ are separated and define $A=g^{-1}(H) \cap M$ and $B=g^{-1}(K) \cap M$. Then for any $x$ $\epsilon A$, there exist open sets $U \in \Re(x)$ and $V \in \Re(f(x))$ such that $(U \times V) \cap K$ $=\varnothing$. Consequently, no point of $U \cap B$ can map into $V$ under $f$. Since $f(M)$ $=f(A) \cup f(B)$ and neither $f(A)$ nor $f(B)$ can be empty, we proceed to show $f(A)$ and $f(B)$ are separated, thereby obtaining a contradiction. Suppose $f(x) \in \mathrm{Cl}(f(B))$ for $x \in A$. Then $f^{-1}(\Re(y)) \cap B$ is a filterbase on the compact set $\mathrm{Cl}(M)$ and, hence, accumulates to some $x_{0} \in \mathrm{Cl}(M)$. Therefore, $x_{0} \epsilon$ $\mathcal{C}^{-1}(f, f(x))$, and, since $f(U \cap B) \cap V=\varnothing$, we have $\mathrm{Cl}\left(f^{-1}(V) \cap B\right) \cap U=\varnothing$. Consequently, $\left\{x, x_{0}\right\} \subset \mathcal{C}^{-1}(f, f(x)) \cap \mathrm{Cl}(M)$ which contradicts the given 
condition of the theorem. We conclude $f(x) \notin \mathrm{Cl}(f(B))$ for every $x \in A$ and, likewise, $f(x) \notin \mathrm{Cl}(f(A))$ for every $x \in B$. This implies $f(M)$ is not connected. Since $f$ is given as a connected function, it must follow that $g$ is connected.

The following Lemma and Theorem give a more workable insight into the condition stated in Theorem 4.1 .

4.2. Lemma. Let $f: X \rightarrow Y$ be a given function and let $A \subset X$. If $\mathcal{C}^{-1}(f \mid A ; y)$ denotes the inverse cluster set of $f \mid A: A \rightarrow Y$ where $A$ has the subspace topology, then $\mathcal{C}^{-1}(f \mid A ; y) \subset \mathcal{C}^{-1}(f ; y) \cap A$ and the equality holds provided $A$ is open.

Proof. The proof consists of the following set relationships:

$$
\begin{aligned}
\mathcal{C}^{-1}(f \mid A ; y) & =\bigcap\left\{\mathrm{Cl}_{A}\left((f \mid A)^{-1}(V)\right): V \in \Re(y)\right\} \\
& =\bigcap\left\{\mathrm{Cl}_{A}\left(f^{-1}(V) \cap A\right): V \in \Re(y)\right\} \\
& \subset \bigcap\left\{\mathrm{Cl}\left(f^{-1}(V)\right) \cap A: V \in \Re(y)\right\} \\
& =\bigcap\left\{\mathrm{Cl}\left(f^{-1}(V)\right): V \in \Re(y)\right\} \cap A \\
& =\rho^{-1}(f ; y) \cap A .
\end{aligned}
$$

Observe that if $A$ is open, the subset relation in the proof is an equality.

4.3. Theorem. Let $f: X \rightarrow Y$ be a given function and consider the following conditions:

(1) For each connected set $M \subset X$ and $x \in M, C^{-1}(f ; f(x)) \cap \mathrm{Cl}(M)=\{x\}$.

(2) For each component $C$ of $X, f \mid C: C \rightarrow f(C)$ is a bijection with a closed graph.

Then (1) implies (2) and if $X$ is locally connected, (1) and (2) are equiv. alent.

Proof. To show (1) implies (2), let $C$ be a component of $X$. Then for each $x \in C$, where $y=f(x)$, we have by Lemma 4.2 and the fact that $C$ is closed,

$$
\rho^{-1}(f \mid C ; y) \subset \mathcal{C}^{-1}(f ; y) \cap C=\mathcal{C}^{-1}(f ; y) \cap \operatorname{Cl}(C)=\{x\} .
$$

Thus, $f \mid C$ is a bijection by Theorem 2.11. Since $\mathcal{C}^{-1}(f \mid C ; y)=f^{-1}(y)$, Theorem 3.2 gives the graph of $f$ closed.

Now assume (2) holds where $X$ is locally connected, $M \subset X$ is connected and $x \in M$. Let $C$ be the component of $X$ containing $\mathrm{Cl}(M)$ and recall that components of locally connected spaces are open so that Lemma 4.2 holds. Then we have

$$
\mathcal{C}^{-1}(f ; f(x)) \cap \mathrm{Cl}(M) \subset \mathcal{C}^{-1}(f ; f(x)) \cap C^{-1}=\mathcal{C}^{-1}(f \mid C ; f(x))=\{x\} .
$$




\section{REFERENCES}

1. M. P. Berri, J. R. Porter and R. M. Stephenson, Jr., A survey of minimal topological spaces, General Topology and its Relations to Modern Analysis and Algebra, III (Proc. Conf., Kanpur, 1968), Academia, Prague, 1971, pp. 93-114. MR $43 \# 3985$.

2. J. Dugundji, Topology, Allyn and Bacon, Boston, Mass., 1966.

3. R. V. Fuller, Relations among continuous and various non-continuous functions, Pacific J. Math. 25 (1968), 495-509. MR 37 \#3536.

4. T. R. Hamlett, Cluster sets in general topology, J. London Math. Soc. (to appear).

5. Paul E. Long, Connected mappings, Duke Math. J. 35 (1968), 677-682. MR $38 \# 2745$.

6. - Conceming semiconnected maps, Proc. Amer. Math. Soc. 21 (1969), 117-118. MR 38 \#5183.

7. J. D. Weston, Some theorems on cluster sets, J. London Math. Soc. 33 (1958), 435-441. MR $20 \# 7109$.

8. Stephen Willard, General topology, Addison-Wesley, Reading, Mass., 1970. MR $41 \# 9173$.

DEPARTMENT OF MATHEMATICS, UNIVERSITY OF ARKANSAS, FAYETTEVILLE, ARKANSAS 72701 